\title{
Control of vessel sprouting by genetic and metabolic determinants
}

\author{
Guy Eelen ${ }^{*}$, Bert Cruys ${ }^{*}$, Jonathan Welti, Katrien De Bock, and Peter Carmeliet
}

Laboratory of Angiogenesis and Neurovascular Link, Vesalius Research Center, Vlaams Instituut voor Biotechnologie (VIB), Department of Oncology, Katholieke Universiteit Leuven (KU Leuven), Herestraat 49, 3000 Leuven, Belgium

\begin{abstract}
Vessel sprouting by endothelial cells (ECs) during angiogenesis relies on a navigating tip cell and on proliferating stalk cells that elongate the shaft. To date, only genetic signals have been shown to regulate vessel sprouting. However, emerging evidence indicates that the angiogenic switch also requires a metabolic switch. Indeed, angiogenic signals not only induce a change in EC metabolism but this metabolic adaptation also co-determines vessel sprouting. The glycolytic activator PFKFB3 regulates stalk cell proliferation and renders ECs more competitive to reach the tip. We discuss the emerging link between angiogenesis and EC metabolism during the various stages of vessel sprouting, focusing only on genetic signals for which an effect on EC metabolism has been documented.
\end{abstract}

\section{Model of vessel sprouting}

Blood vessels rapidly switch from a state of quiescence to active sprouting in response to pro-angiogenic stimuli. Specific EC subtypes orchestrate vessel sprouting. Navigating tip cells (see Glossary) lead the vessel branch at the vascular forefront, while proliferating stalk cells elongate the shaft in the new sprout [1]. Neighboring sprouts anastomose through interactions between filopodia on their respective tip cells to form a new and perfused vessel branch. ECs then regain their quiescent state (phalanx cells) and are stabilized by the coverage of perivascular mural pericytes [2]. Instead of providing a complete survey of all known genetic molecules known to regulate vessel sprouting (for which we refer to more historical overviews [3-6]), we limit our overview here to molecules with known effects on EC metabolism. We first describe the metabolic pathways that ECs use to generate energy.

\section{Energy generation in ECs}

Glucose metabolism

In contrast to other healthy cells [7,8], ECs rely heavily on glycolysis to generate energy (Figure 1) [8-10]. The glycolysis rate in ECs is comparable to the rate measured

\footnotetext{
Corresponding author: Carmeliet, P. (peter.carmeliet@vib-kuleuven.be).

Keywords: angiogenesis; tip cell; branching; filopodia; glycolysis; PFKFB3.

These authors contributed equally to this work.

1043-2760/\$ - see front matter

(C) 2013 Elsevier Ltd. All rights reserved. http://dx.doi.org/10.1016/j.tem.2013.08.006
}

in various tumor cells [8]. Glycolysis is essential for ECs to maintain homeostasis because nearly complete inhibition of glycolysis with the glycolysis inhibitor 2-deoxy-D-glucose (2-DG) induces cell death [8]. Notably, ECs rely minimally on glucose oxidation and mitochondrial respiration for ATP production $[8,10]$. Nonetheless, mitochondria represent a bioenergetic reserve to which ECs appeal under stressed conditions [11].

Upon induction of sprouting by growth factors such as vascular endothelial growth factor (VEGF), quiescent

\section{Glossary}

Anastomosis: the process in which a tip cell meets and fuses with another tip cell or a pre-existing blood vessel to form a lumenized loop that allows blood flow.

Filopodia: thin $(0.1-0.3 \mu \mathrm{m})$ long $(10 \mu \mathrm{m})$ finger-like actin-rich plasma membrane protrusions composed of parallel bundles of filamentous actin important in cell migration, adhesion to the extracellular matrix, guidance towards chemoattractants, cell-cell signaling, embryonic development, and wound healing.

Glycocalyx: an interconnected gel-like network of membrane-bound proteoglycans and glycoproteins coating the luminal surface of ECs. This layer is involved in the mechano-transduction of shear stress, the adherence of blood cells, vascular permeability, and vessel wall homeostasis.

Glycolysis: the breakdown of glucose to pyruvate in 10 consecutive cytoplasmic reactions to generate energy (ATP) and reducing power (NADH). Hexosamine biosynthesis pathway (HBP): the metabolic pathway of which the rate-limiting step is the transfer of the glutamine amido group to fructose-6phosphate. Ultimately, glucosamine-6-phosphate, the key precursor for $\mathrm{O}$ - and $\mathrm{N}$-linked protein glycosylation, is generated.

Lamellipodia: thin $(0.1-0.2 \mu \mathrm{m})$ sheet-like protrusions behind the protruding cell edge consisting of a densely branched filamentous actin network and that are important in cell migration, substrate adhesion, macropinocytosis, and phagocytosis.

Pentose phosphate pathway (PPP): the metabolic pathway that converts glucose-6-phosphate to ribose-5-phosphate, a key intermediate of nucleotide synthesis, with production of NADPH, and is important for several anabolic processes and redox homeostasis.

Pericytes: (peri, around; cyte, cell) mural cells that cover blood microvessels and share the vascular basement membrane. They provide support, ensure vessel stability, and regulate permeability by direct physical contact and paracrine signaling with ECs.

Phalanx cells: specialized ECs that are quiescent, lack filopodia, and are aligned in a smooth tight cobblestone monolayer to ensure optimal blood flow and stable perfusion of the surrounding tissues.

6-Phosphofructo-2-kinase/fructose-2,6-bisphosphatase 3 (PFKFB3): a key regulatory bifunctional enzyme of the glycolytic flux. It has a high kinase:phosphatase ratio ( 740:1) and as such generates high amounts of fructose-2,6bisphosphate, the most potent allosteric activator of the rate-limiting glycolytic enzyme phosphofructokinase-1 (PFK-1).

Spheroid assay: the use of in vitro established 3D EC aggregates to study EC sprouting.

Stalk cells: specialized ECs that form the stalk of vascular sprouts. Stalk cells trail behind tip cells, proliferate to elongate the stalk, and form a lumen. They also prevent retraction of ECs.

Tip cells: specialized ECs located at the tip of the vascular sprout. These cells are highly polarized, extend numerous filo- and lamellipodia with which they probe the environment, and are key to both anastomosis and migration because they direct the sprout. 


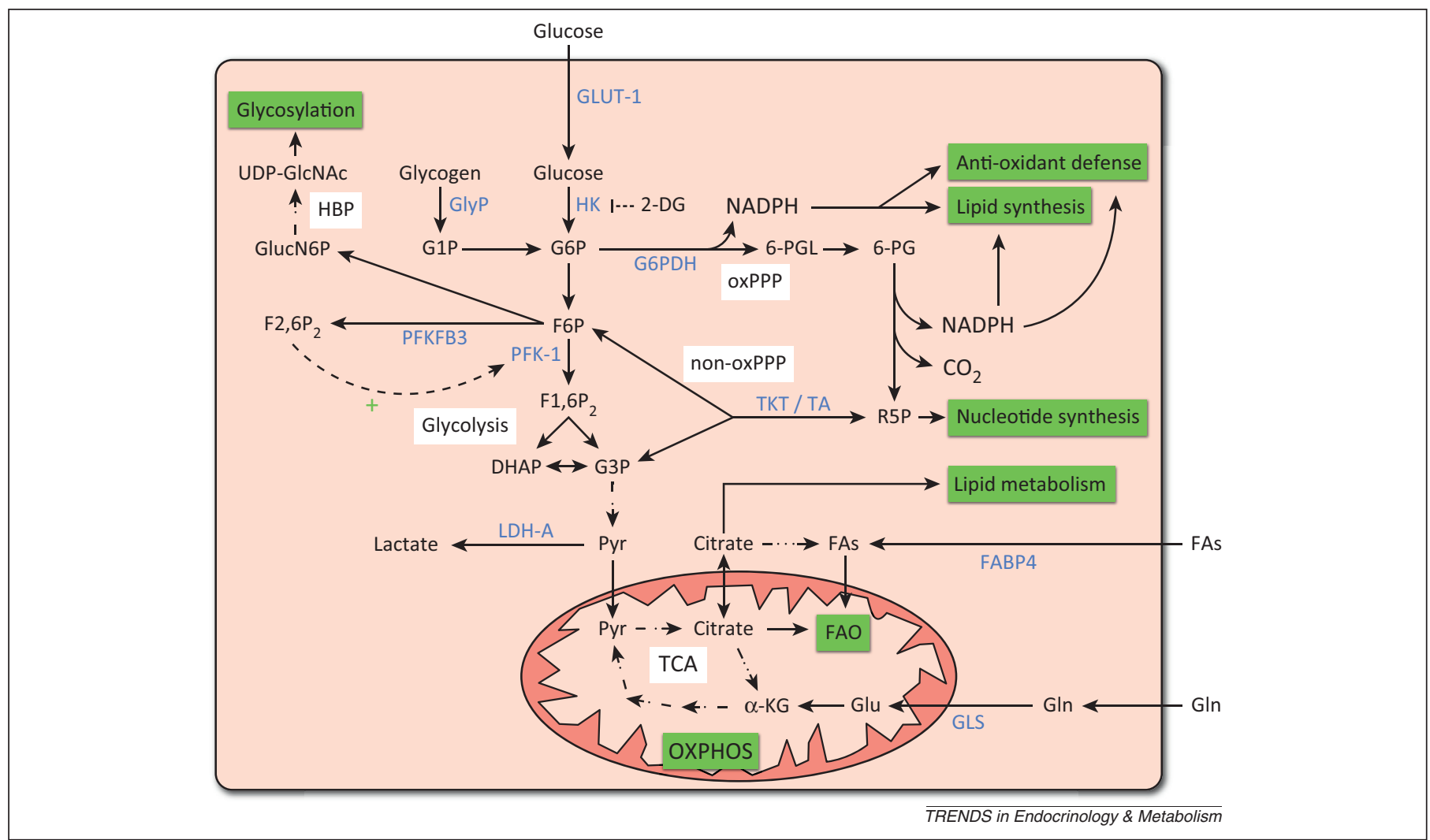

Figure 1. Endothelial cell (EC) metabolism. Schematic overview of the principal metabolic pathways and key enzymes in ECs. Glucose is taken up via the GLUT-1 transporter and subsequently phosphorylated by hexokinase. Quiescent ECs mainly rely on glycolysis to generate the energy needed to perform their functions, whereas sprouting ECs even further upregulate their glycolysis rate. Glycolysis intermediates are shunted into side-branches for anti-oxidant defense and lipid synthesis (oxPPP) as well as nucleotide synthesis (both oxPPP and non-oxPPP) and glycosylation purposes (HBP). Upon glucose scarcity, intracellular reserves can be broken down to sustain the rate of glycolysis. ECs also use FAs and glutamine as energy sources via FAO and glutaminolysis respectively. The latter is required to maintain the functionality of the TCA cycle (anaplerosis) as TCA cycle intermediates (e.g., citrate) exit for anabolic purposes. Abbreviations: 2-DG, 2-deoxy-D-glucose; DHAP, dihydroxyacetone phosphate; $\mathrm{F} 1,6 \mathrm{P}_{2}$, fructose-1,6-bisphosphate; $\mathrm{F} 2,6 \mathrm{P}_{2}$, fructose-2,6-bisphosphate; F6P, fructose-6-phosphate; FABP4, fatty acid binding protein 4; FAs, fatty acids; FAO, fatty acid oxidation; G1P, glucose-1-phosphate; G3P, glyceraldehyde-3-phosphate; G6P, glucose-6-phosphate; G6PDH, G6P dehydrogenase; GLS, glutaminase; GIn, glutamine; Glu, glutamate; GlucN6P, glucosamine-6-phosphate; GLUT-1, glucose transporter 1; GlyP, glycogen phosphorylase; HBP, hexosamine biosynthetic pathway; HK, hexokinase; $\alpha$ KG, $\alpha$-ketoglutarate; LDH-A, lactate dehydrogenase A; NADPH, nicotinamide adenine dinucleotide phosphate; (non-)oxPPP, (non-)oxidative branch of the pentose phosphate pathway; OXPHOS, oxidative phosphorylation; 6-PG, 6-phosphogluconate; 6-PGL, 6-phosphogluconolactone; PFK-1, phosphofructokinase-1; PFKFB3, 6phosphofructo-2-kinase/fructose-2,6-bisphosphatase-3; Pyr, pyruvate; R5P, ribulose-5-phosphate; TA, transaldolase; TCA, tricarboxylic acid; TKT, transketolase; UDPGlcNAc, uridine diphosphate $N$-acetylglucosamine.

ECs increase their glycolysis rate by upregulating the expression of, amongst others, glucose transporter 1 (GLUT-1: facilitates the transport of glucose across the plasma membrane), lactate dehydrogenase-A (LDH-A: converts pyruvate and $\mathrm{NADH}$ to L-lactate and $\mathrm{NAD}^{+}$), and 6-phosphofructo-2-kinase/fructose-2,6-bisphosphatase-3 (PFKFB3) $[8,9,12,13]$. PFKFB3 synthesizes fructose-2,6-bisphosphate $\left(\mathrm{F} 2,6 \mathrm{P}_{2}\right)$, an allosteric activator of phosphofructokinase-1 (PFK-1) and the most potent stimulator of glycolysis [14]. Reducing glycolysis by PFKFB3 gene silencing in ECs in vitro decreases EC migration and proliferation, and impairs vessel sprouting in EC spheroid assays. Furthermore, PFKFB3 gene inactivation in ECs in vivo impedes vessel sprouting, branching, and outgrowth [8].

In view of the exposure of ECs to oxygen in the blood, the importance of glycolysis in ECs might seem paradoxical given that oxidative phosphorylation (OXPHOS) is more efficient in generating ATP (approximately 20-fold more ATP per glucose molecule [15]). However, this preference can be explained by several reasons. First, endothelial oxygen consumption would decrease the availability for trans-endothelial transport to perivascular cells. Second, the low oxygen-dependence of ECs might prepare them for sprouting in hypoxic tissues. As such, ECs are able to quickly sprout without the need to adapt to fluctuating oxygen levels. This is further strengthened by the fact that oxygen is more limiting than glucose in the tumor interstitium (compared to glucose, oxygen has a higher diffusion coefficient, but lower solubility and therefore also lower available tissue concentration, resulting in a shorter diffusion distance and steeper concentration gradient from the vessel to the tissue [16-18]), and because ECs are only susceptible to hypoxia when glucose is limiting [19]. Third, lower oxidative metabolism results in less oxidative stress. Fourth, when glucose availability is unlimited, the glucose uptake and glycolysis rates can be elevated to equal or even exceed the ATP production by OXPHOS $[10,15]$. Finally, to sustain cell division and migration, glycolysis intermediates can be shunted to side-branches such as the pentose phosphate pathway (PPP) and the hexosamine biosynthetic pathway (HBP).

The PPP is essential for nucleotide, lipid, and aromatic amino acid synthesis as well as for anti-oxidant protection, whereas the HBP is considered a nutrient sensor important in protein glycosylation (Figure 1). The first and ratelimiting step of the oxidative part of the PPP (oxPPP) is 
catalyzed by glucose-6-phosphate (G6P) dehydrogenase (G6PDH), the plasma membrane localization and activity of which is regulated by Src-mediated tyrosine phosphorylation, and which plays a role in VEGF-mediated phosphorylation of Akt, VEGFR2, and eNOS, and EC responses such as migration, proliferation, and tube formation $[20,21]$. This is exemplified by a decrease in EC viability and migration upon G6PDH inhibition by either a pharmacological compound [22] or by high glucose levels [23]. Inhibition of transketolase, an enzyme involved in the nonoxidative part of the PPP (non-oxPPP), also reduces EC viability and migration [22]. Interestingly, inhibition of the non-oxPPP has a more pronounced effect on EC viability and migration than inhibition of the oxPPP, suggesting that the reversible non-oxPPP compensates for the reduced nucleotide synthesis via the oxPPP [22]. However, it is worth mentioning that the relative importance of the oxPPP in vitro might have been underestimated because of the high glucose concentrations used in many studies, which can suppress this branch [23]. Hence, both arms may be important, but in different metabolic contexts.

Cells store energy in glycogen reservoirs that can be used when extracellular glucose levels are insufficient to meet the energy demand (Figure 1). VEGF signaling and glucose deprivation induce glycogenolysis, which is the breakdown of glycogen to G6P in ECs [22]. Further, pharmacological inhibition of glycogen phosphorylase (and thus of glycogenolysis) reduces EC viability and migration [22]. Although glycogenolysis-derived G6P only has a limited contribution to glycolysis and energy production [24], it may have more importance in glycolysis side-branches such as the oxPPP. This has recently been shown to be the case for tumor cells [25], but similar evidence in ECs is still lacking. Overall, it is not yet clear which role glycogen has in vascular sprouting.

\section{Other metabolic pathways}

In addition to glucose, ECs also use glutamine and fatty acids as fuels (Figure 1). ECs are well-equipped to take up and metabolize glutamine [26,27], a conditionally essential amino acid [28]. Glutaminase catalyzes the conversion of glutamine to glutamate, the first step of glutaminolysis that yields $\alpha$-ketoglutarate. The latter is used in the tricarboxylic acid (TCA) cycle to replace TCA cycle intermediates that have exited for anabolic purposes, thus sustaining the functionality of the TCA cycle (anaplerosis) [28]. In addition, glutamine is a key nitrogen source [28] and contributes to improved viability of ECs under oxidative stress [29]. The glutaminase activity of ECs has been reported to be high [10] and its inhibition induces EC senescence [30].

ECs can also obtain energy via the oxidation of fatty acids from intra- and extracellular sources [31] (Figure 1). Endothelial fatty acid oxidation (FAO) is increased in vitro upon glucose deprivation through activation of AMP-activated protein kinase (AMPK), and upon stimulation with carnitine [31] which is required for mitochondrial import of fatty acids. VEGF-B is a poor angiogenic factor with relatively restricted activity on coronary vessels, but VEGF-B signaling via VEGF receptor 1 (VEGFR1)/neuropilin 1 increases the uptake and trans-endothelial transport of fatty acids in ECs [32], but without influencing FAO in ECs [33]. VEGF induces the expression levels of fatty acid binding protein 4 (FABP4). This protein is involved in uptake and trafficking of intracellular fatty acids, and knockdown of FABP4 reduces EC proliferation [34], indicating the importance of lipid metabolism.

\section{Sprouting in a metabolically taxing environment: tip versus stalk cells Genetic signals}

In response to hypoxia, the key angiogenic signal VEGF is secreted in an attempt to restore tissue oxygenation by promoting vessel sprouting. When VEGF reaches the vascular front it binds to VEGF receptor 2 (VEGFR2) on ECs. The EC exposed to the highest level of VEGF is selected to become a tip cell. The current model of vascular branching is based on the coordinated actions of migrating tip cells, taking the lead at the vascular front, and proliferating stalk cells elongating the newly developing branch [1], with the fate of both cell types being dynamically interchangeable rather than being permanently fixed [35] (Figure 2A).

The specification of tip versus stalk cells is dictated by VEGF-Notch signaling. VEGFR2-activated tip cells express the Notch ligand Delta-like 4 (Dll4), which binds to Notch receptors (single-pass transmembrane receptor proteins) in neighboring ECs. This results in the release of the receptor intracellular domain from the membrane, Notch intracellular domain (NICD), which translocates to the nucleus and regulates transcription of target genes [36]. As a result, VEGFR2 expression is lowered whereas levels of the VEGF trap VEGFR1 increase, which renders ECs less responsive to VEGF, thus out-competing their ability to become a tip cell and promoting a stalk cell fate (Figure 2A). Dynamic tip cell-stalk cell fate interchange or 'cell shuffling' occurs at the vascular front to allow the ECs with the highest VEGFR2 and lowest VEGFR1 expression levels to occupy the tip position to ensure maximal fitness of leading the sprout $[35,37]$. There are many more molecules regulating tip versus stalk cell behavior, such as for instance VEGF-C/VEGFR3, the angiopoietins, the TGF- $\beta$ / BMP9 receptor, and others [1], but these signals are not known to affect EC metabolism in physiological conditions.

\section{Metabolic signals}

Recent findings indicate that VEGF increases glycolysis in ECs by upregulating PFKFB3 mRNA and protein levels before ECs start to proliferate, whereas Dll4-induced prostalk Notch signaling induces the opposite effect [8], implying that tip cells have higher glycolysis rates (Figure 2B). Nonetheless, glycolysis is also essential for stalk cell functions. Stalk cells actively proliferate and therefore must generate high amounts of ATP and macromolecules that are derived from metabolic pathways, such as the PPP, that branch off from the main glycolytic pathway [7] (Figure 2C). However, Notch inhibits EC proliferation in vitro. Hence, the growth-inhibitory function of Notch signaling must be overridden in vivo. Indeed, Notch in stalk cells induces the expression of the Notchregulated ankyrin repeat protein (Nrarp), which in turn enhances Wnt signaling to promote stalk cell proliferation and vessel stability [38]. 

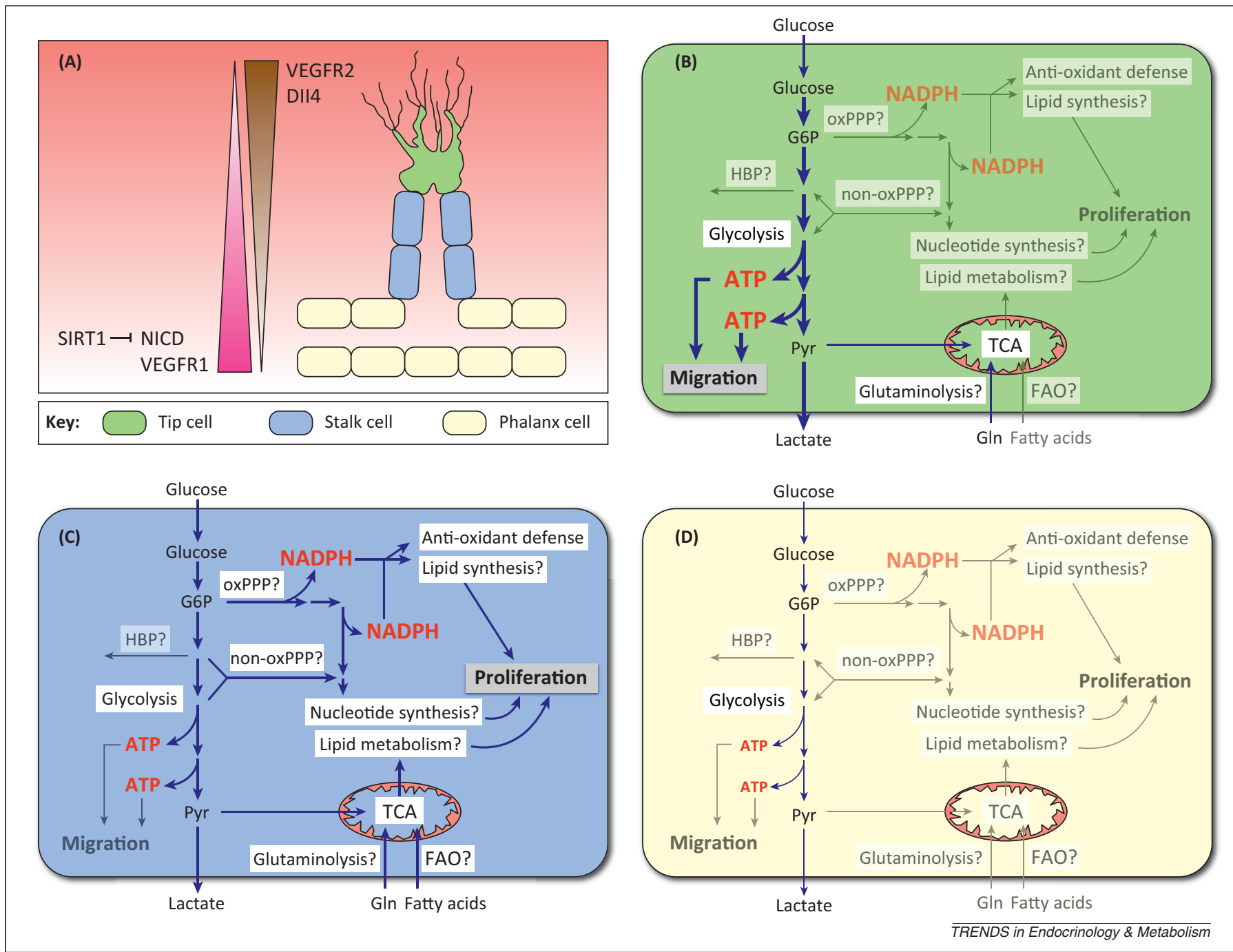

Figure 2. Genetic and metabolic determinants of tip, stalk and phalanx cell behavior. (A) Schematic representation of a vascular sprout with tip cell (green), stalk cell (blue) and phalanx cell (yellow) and key genetic fate-determining factors. Upon sensing a VEGF gradient (red background) the tip cell induces DII4 expression to activate Notch signaling in the adjacent stalk cells (NICD release) and subsequently to decrease VEGFR2 and increase VEGFR1 levels to reduce VEGF sensitivity. SIRT1 translates energy stress into NICD degradation resulting in tip cell behavior. (B-D) Different types of metabolism characterize the different EC subtypes; unknown metabolic changes are indicated by question marks. For their migratory behavior, tip cells require large amounts of ATP. These are generated primarily by the high glycolytic flux (B). Stalk cells divide to elongate the newly formed sprout and consequently require building blocks for rapid macromolecular synthesis. To obtain these precursors, NADPH is generated in the oxPPP, nucleotides are synthesized via the non-oxPPP and/or oxPPP, and fatty acids and ATP are obtained by combining glycolysis and likely also anapleurotic glutaminolysis (C). Phalanx cell metabolism ensures cellular homeostasis and provides energy for vital functions (D). Abbreviations: DII4, Delta-like 4; FAO, fatty acid oxidation; G6P, glucose-6-phosphate; Gln, glutamine; HBP, hexosamine biosynthetic pathway; NADPH, nicotinamide adenine dinucleotide phosphate; NICD, Notch intracellular domain; (non-)oxPPP, (non-)oxidative branch of the pentose phosphate pathway; Pyr, pyruvate; SIRT1, Sirtuin1; TCA, tricarboxylic acid; VEGF, vascular endothelial growth factor; VEGFR, VEGF receptor.

Remarkably, overexpression of PFKFB3 overcomes the pro-stalk activity of Notch signaling and promotes stalk cells to adopt a tip cell behavior, both in EC spheroid sprouting assays in vitro and in zebrafish vascular branching in vivo, whereas silencing of PFKFB3 causes opposite effects in baseline conditions and upon inhibition of Notch signaling [8]. These findings are intriguing because no other genetic signal is so far known to be able to overrule the pro-stalk activity of Notch. Notably, over- or underexpression of PFKFB3 in baseline conditions or in conditions favoring tip cell behavior (upon inhibition of Notch signaling), or stalk cell behavior (upon activation of Notch signaling), did not alter the molecular signature of genes enriched in either tip or stalk cells, indicating that PFKFB3 did not affect tip and stalk cells through regulation of tip/stalk cell signals [8]. Instead, these studies show for the first time that metabolism (in this case, PFKFB3-driven glycolysis) controls vessel branching in parallel to genetic signals (Figure 3).

The metabolic status of the milieu into which new vessels branch out complements the above mechanisms via a feedback loop involving the $\mathrm{NAD}^{+}$-dependent deacetylase Sirtuin 1 (SIRT1). Upon sensing energy/fuel stress, SIRT1 activity increases to deacetylate and to pre-destine NICD for proteasomal degradation [39] (Figure 2A). These findings might imply that the subsequent lowering of the Notch-driven pro-stalk activity allows SIRT1 to direct tip cells towards fuel-rich areas. Conversely, ECs with reduced SIRT1 activity fail to sense nutrient stress adequately and are sensitized to pro-stalk Notch signaling, resulting in impaired vessel growth [40]. 
(A)

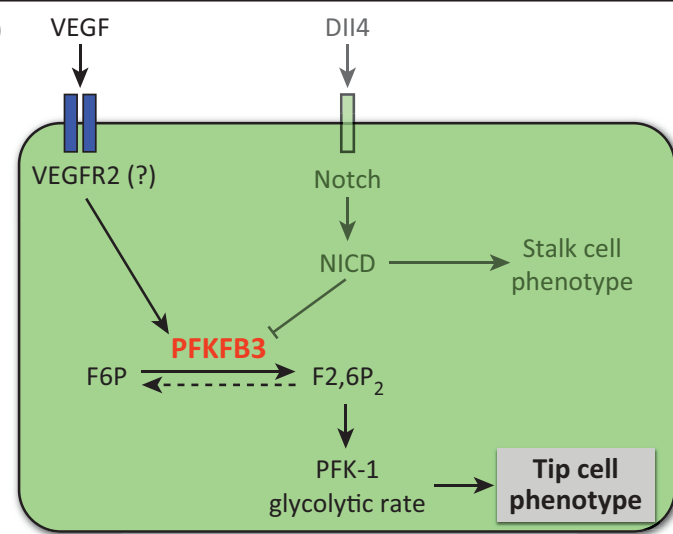

(B)

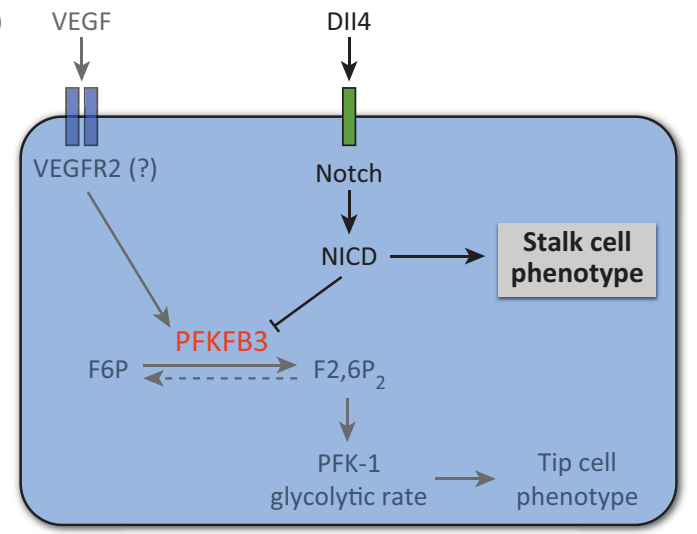

(C)

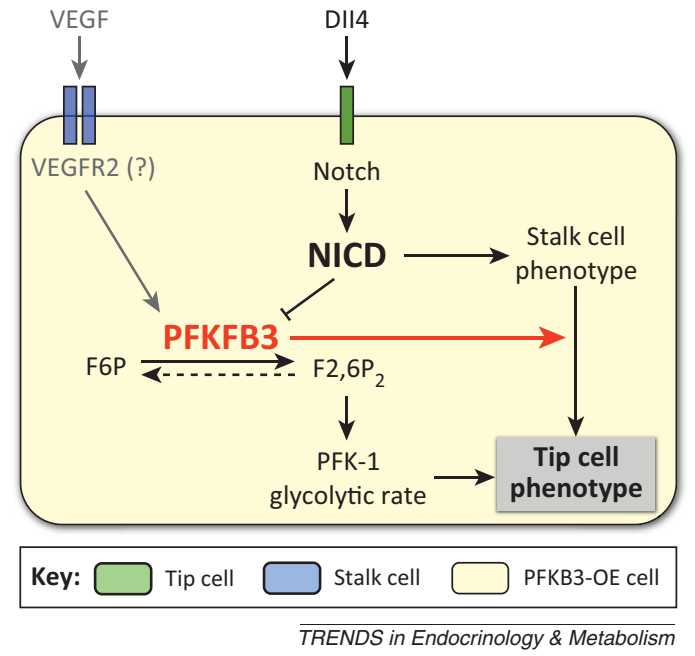

Figure 3. Metabolic switching between tip and stalk cells. (A) Metabolic glycolytic signature of tip cells: tip cells are known to have high VEGFNEGFR2 signaling. Activation of VEGFR2 by VEGF upregulates PFKFB3 levels. PFKFB3 produces $F 2,6 P_{2}$ which is the most potent stimulator of PFK-1, a rate-limiting enzyme of the glycolytic pathway. Consequently, the glycolysis rate is elevated which renders ECs more competitive to reach the tip. By contrast, DII4/Notch signaling, releasing NICD, reduces glycolysis by lowering PFKFB3 expression, but this signaling pathway is less active in tip cells. (B) Metabolic glycolytic signature of stalk cells: stalk cells are known to have high DII4/Notch signaling. Activation of Notch by DII4 lowers PFKFB3-driven glycolysis, thereby reducing competitiveness for the tip position. (C) Overexpression of PFKFB3 induces switching from the stalk to the tip cell phenotype: Overexpression of PFKFB3 in ECs, instructed to adopt a stalk cell phenotype by overexpression of NICD, is capable of overcoming the stalk cell phenotype and increasing the competitiveness of these cells for the tip position. Abbreviations: DII4, Deltalike 4; $\mathrm{F}_{2}, 6 \mathrm{P}_{2}$, fructose-2,6-bisphosphate; $\mathrm{F} 6 \mathrm{P}$, fructose-6-phosphate; NICD, Notch intracellular domain; PFK-1, phosphofructokinase-1; PFKFB3, 6-phosphofructo-2kinase/fructose-2,6-bisphosphatase-3; PFKFB3-OE, PFKFB3-overexpressing; VEGF, vascular endothelial growth factor; VEGFR2, VEGF receptor 2 (the question mark indicates that VEGFR2 probably mediates the VEGF response, but this needs to be proven in the future).
The HBP pathway is another nutrient sensing pathway. Inhibition of this pathway by 2-DG impedes angiogenesis, which can be rescued by the $N$-linked glycosylation precursor mannose [41]. It is therefore tempting to speculate that the HBP in ECs serves to translate nutrient stress into adjusting blood vessel supply by generating $N$-acetylglucosamine that post-translationally modifies and activates key angiogenic receptors. Indeed, VEGFR2 function requires $\mathrm{N}$-glycosylation [42]. Also noteworthy is the evidence that the Notch response to the ligands Dll4 and Jagged1 depends on the level of glycosylation of its extracellular domain [43].

Metabolites can also control angiogenesis by acting as bona fide signaling molecules. An example is the glycolysis product lactate, which is taken up by ECs via the monocarboxylate transporter 1 (MCT1), and which mainly has a signaling role because it seems to be only marginally metabolized [44], at least at normal extracellular glucose levels [24]. Once taken up by ECs, lactate competitively inhibits the oxygen-sensing prolyl hydroxylase domain protein 2 (PHD2), resulting in activation of hypoxia-inducible factor $1 \alpha(\mathrm{HIF}-1 \alpha)$ and a secondary increase in VEGFR2 and basic fibroblast growth factor expression [45]. PHD2 inhibition by lactate also triggers IкB $\alpha$ degradation, releasing the transcription factor NF- $\mathrm{kB}$ to induce the expression of the pro-angiogenic interleukin-8 [44]. Lactate also engages the PI3K/Akt pathway via upregulation of the ligands and subsequent activation of three receptor tyrosine kinases: Axl, the angiopoietin receptor Tie2, and VEGFR2 [46]. Moreover, lactate signaling induces VEGF expression [47] and reduces post-translational poly-ADP ribosylation of VEGF, thereby making it more potent [48]. Lactate also promotes collagen and proteoglycan matrix deposition [49], and stimulates EC migration and capillary tube formation [44,49], although it remains to be defined if matrix deposition induced by lactate is causally linked to vessel sprouting. Finally, lactate induces pro-angiogenic reactive oxygen species in ECs [44].

\section{Compartmentalization of glycolysis in F-actin-rich lamellipodia}

In ECs, both filopodia and lamellipodia have typical actin networks (branched actin for lamellipodia versus aligned filamentous actin for filopodia) that can be assembled within minutes after growth factor stimulation [50]. The highly dynamic nature of these structures relies on active actin cytoskeleton remodeling and requires rapid generation and instantaneous availability of large amounts of ATP; actin polymerization for example is tightly linked to ATP hydrolysis [51-53]. Nevertheless, both filopodia and lamellipodia are devoid of mitochondria [8], likely because mitochondria (ranging in size from 0.5 to over $4 \mu \mathrm{m}$ ) cannot spatially fit into the narrow filopodia and lamellipodia $(\sim 250 \mathrm{~nm}$ wide and high, respectively) [54] (Figure 4A,C,D).

It is unlikely that sufficient amounts of ATP reach the very tip of the filopodia (up to $10 \mu \mathrm{m}$ in length) sufficiently fast by diffusion from the distant perinuclear mitochondrial network; but, even so, it is conceivable that the continuous protrusion and retraction of filopodia and lamellipodia locally generates such a high ATP demand that ATP levels 

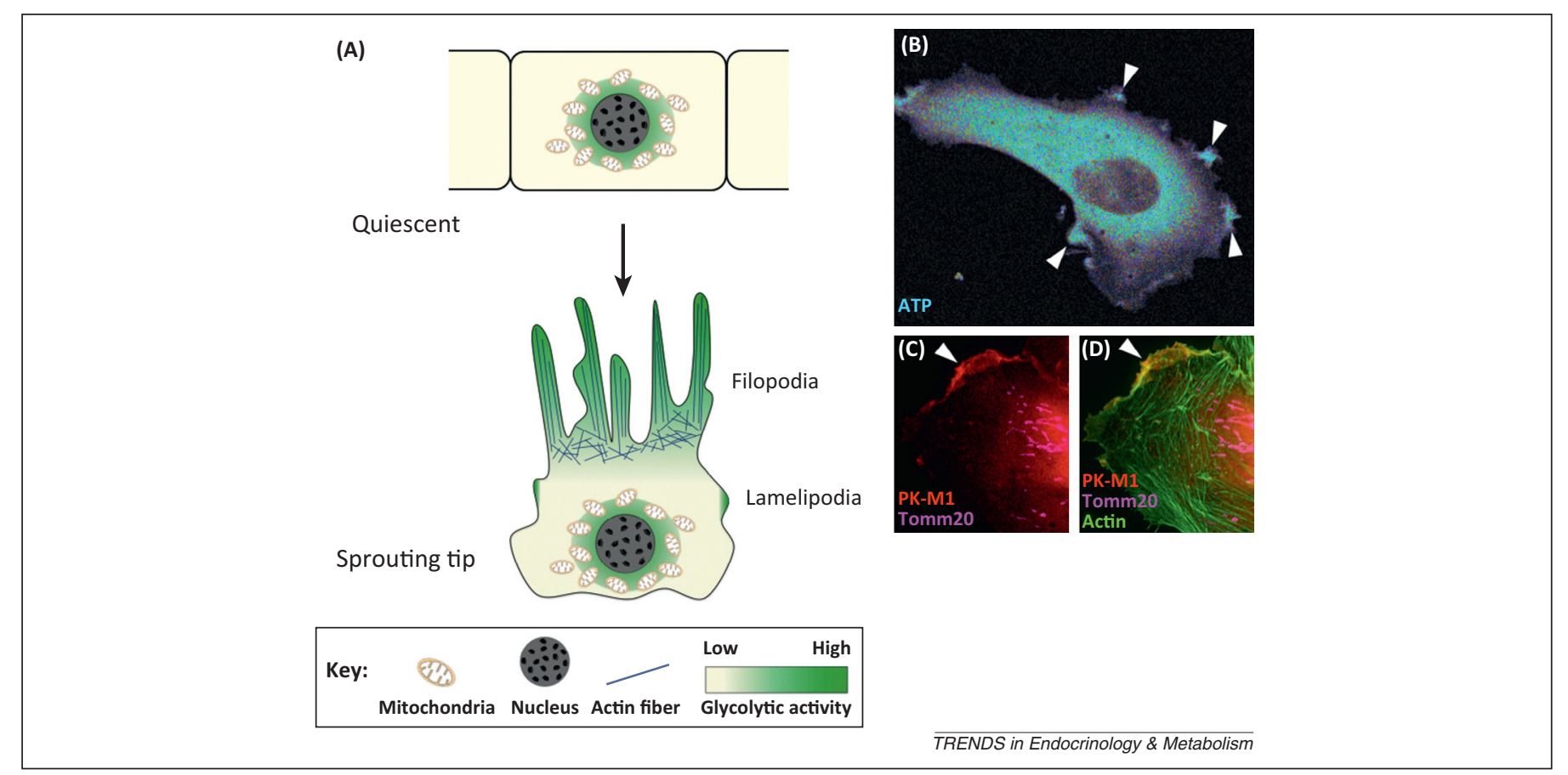

Figure 4. Compartmentalization of glycolysis with F-actin. (A) Schematic representation of a quiescent and a tip cell with mitochondria in the perinuclear region but, in the case of the tip cell, not in filopodia or lamellipodia in which glycolytic activity is compartmentalized with F-actin. (B) Imaging of intracellular ATP levels in endothelial cells (ECs) with a GO-Ateam ATP fluorescence resonance energy transfer (FRET) probe showing the presence of ATP in lamellipodia (white arrowheads). (C,D) Presence of

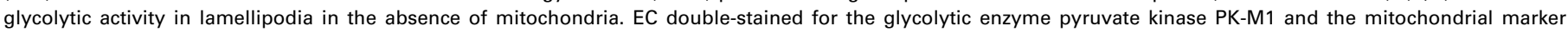
Tomm20 (lamellipodia indicated by white arrowhead) (C) and merged with actin staining (D).

throughout the whole cell body might become compromised during active lamellipodial and filopodial activity. To avoid this, ECs compartmentalize glycolysis with the actin skeleton in cellular protrusions, mediating EC motility [8]. Various glycolytic enzymes contain actin-binding sites, and binding to $\mathrm{F}$-actin enhances their activity [55]. In migrating ECs, both PFKFB3 and other glycolytic enzymes - such as phosphoglycerate kinase and the pyruvate kinase isoenzyme that generates ATP (PK-M1), rather than the isoenzyme involved in biosynthesis of macromolecules (PK-M2) - are enriched in the membrane ruffles at the leading front of lamellipodia, and co-concentrate with $\mathrm{F}$ actin in these structures [8] (Figure 4B-D). Co-immunoprecipitation experiments confirmed the PFKFB3-actin interaction and showed that the fraction of PFKFB3 bound to actin increases during EC motility [8]. Localizing glycolysis-driven ATP production at the site of high ATP demand during motility prevents the cell from succumbing to overall ATP depletion. Of note, compartmentalization of glycolysis in motile structures originated early in evolution: for example, flagella in various microorganisms and tentacles on members of the basal metazoan Hydra genus [56,57]. Noteworthy in this respect, astrocytes localize glycogen depots in their lamellipodial and filopodial extensions, and glycolysis and glycogenolysis are essential for astrocytic responses to increasing energy demand [58].

\section{Phalanx cells: quiescent again}

\section{Genetic signals}

ECs resume a quiescent state once the new vessel branch is formed, and then adopt a cobblestone-like appearance that resembles the ancient Greek military phalanx formation hence the term 'phalanx cells' [59]. These cells have few filopodia, are non-proliferating, survive for years, migrate poorly, and have an attenuated response to VEGF. Phalanx cells express elevated levels of VEGFR 1 to diminish the pro-angiogenic signaling of VEGF [59] (Figure 2A). Elevated signaling through Notch and the Tie2 receptor in response to elevated levels of angiopoietin-1 between ECs in trans additionally promotes EC quiescence [60,61].

Phalanx cells form a tight barrier and their monolayer apposition allows conduction of blood flow, which by itself is an important regulator of EC quiescence [1]. Vessel stabilization is further enhanced by the deposition of a basement membrane (BM) around quiescent ECs; this is in part due to the fact that the BM component laminin $\alpha 4$ reduces tip cell specification by inducing Notch signaling $[62,63]$. The expression of vascular endothelial cadherin (VE-cadherin) and neuronal cadherin (N-cadherin) in quiescent ECs is crucial for cell-cell adhesion and optimal endothelial barrier function, which are required for controlled exchange of fluids and solutes between the bloodstream and the neighboring tissue $[64,65]$. Lowering of proangiogenic signals and increasing pro-quiescence molecules promote ECs quiescence.

\section{Metabolic signals}

Less is known about the metabolic changes that precede or accompany the EC's switch to quiescence. Interestingly, cell quiescence is often characterized by a higher level of metabolism than previously anticipated [15]. Quiescent ECs lower their glycolysis rate only by $40 \%$ - the proportion of total glycolysis that is required for proliferation and migration [8] (proliferation has been estimated to require a $30 \%$ increase in ATP production over quiescence [66]). The remaining $60 \%$ of glycolysis in quiescent ECs ensures 
cellular homeostasis and provides the necessary energy for vital functions such as safeguarding barrier functions, matrix deposition, and glycocalyx production [1,67] (Figure 2D).

\section{Concluding remarks and future perspectives}

Over the past decade the angiogenesis field has gained an enormous insight into the molecular players that control the formation of vessels in physiological and pathological conditions. Metabolism only recently entered the field of angiogenesis as an important player. Although there are some published data on the role of separate metabolic pathways in ECs in vitro, a unifying view on how major metabolic pathways converge in in vivo vessel branching is almost nonexistent. Recent findings on how PFKFB3-driven glycolysis determines vessel branching have only shown the proverbial tip of the iceberg, and numerous outstanding questions remain to be addressed (Box 1). For example, a 'metabolo-map' of ECs in quiescence versus sprouting state is largely missing, and other pathways besides glycolysis have been only minimally studied. Furthermore, it is currently unknown how other branching signals (aside from VEGF and Notch) control metabolism and vice versa. The importance of metabolic communication between ECs and other cell types, in normal tissues as well as in the tumor microenvironment, remains unclear. In addition, it is crucial to understand how the metabolic adaptations of growing tumor vessels differ from those of rapidly proliferating cancer cells because this will influence our therapeutic targeting strategies. Another outstanding question is whether the definition of an underlying metabolic shift that ECs undergo from quiescence to angiogenesis will allow the development of new 'anti metabolic anti angiogenic' agents, and whether targeting EC metabolism could therefore provide a valuable alternative to overcome resistance to currently approved anti angiogenic therapies, without causing unacceptable toxicity. This is a timely question given that novel anti angiogenic strategies with fundamentally new mechanisms will likely aid in overcoming toxicity and resistance issues of current anti angiogenic therapies [3]. The generation of EC-specific metabolic knockout animals, as well as the continuous improvement of techniques to assess metabolic fluxes of ECs in quiescent and growing vessels in vivo, will be required to answer all these intriguing questions.

\section{Box 1. Outstanding questions}

- What are the metabolic properties of ECs in quiescent as well as sprouting conditions?

- What other pathways in addition to glycolysis are involved in sprouting?

- How do other branching signals (aside from VEGF and Notch) control metabolism, and vice versa?

- How important is metabolic communication between ECs and other cell types in normal tissues as well as in the tumor microenvironment?

- In which sense do the metabolic adaptations of growing tumor vessels differ from those of rapidly proliferating cancer cells, and will this influence our therapeutic targeting strategies?

- Will targeting EC metabolism provide a valuable alternative for overcoming resistance to currently approved anti angiogenic therapies, without causing unacceptable toxicity?

\section{Disclaimer statement}

P.C. declares to be named as inventor on patent applications claiming subject matter related to the results described in this paper.

\section{Acknowledgments}

We apologize to all colleagues whose work was not cited in this review because of space limitations and journal guidelines to cite only recent papers (or reviews for older publications). J.W. is a postdoctoral fellow of the Marie Curie Foundation; K.D.B. is a postdoctoral fellow of the Research Foundation-Flanders (FWO) and is currently an academic staff member at the Department of Kinesiology (KU Leuven). B.C. is funded by the Institution of Research and Innovation (IWT). The work of P.C. is supported by a Federal Government Belgium grant (IUAP P7/03), longterm structural Methusalem funding by the Flemish Government, the Belgian Science Fund (FWO grants), the Foundation Leducq Transatlantic Artemis Network, a European Research Council (ERC) Advanced Research Grant (EU-ERC269073), and the AXA Research Fund.

\section{References}

1 Potente, M. et al. (2011) Basic and therapeutic aspects of angiogenesis. Cell 146, 873-887

2 Siekmann, A.F. et al. (2013) The tip cell concept 10 years after: new players tune in for a common theme. Exp. Cell Res. 319, 1255-1263

3 Carmeliet, P. and Jain, R.K. (2011) Molecular mechanisms and clinical applications of angiogenesis. Nature 473, 298-307

4 Chung, A.S. and Ferrara, N. (2011) Developmental and pathological angiogenesis. Annu. Rev. Cell Dev. Biol. 27, 563-584

5 Eilken, H.M. and Adams, R.H. (2010) Dynamics of endothelial cell behavior in sprouting angiogenesis. Curr. Opin. Cell Biol. 22, 617-625

6 Saharinen, P. et al. (2011) VEGF and angiopoietin signaling in tumor angiogenesis and metastasis. Trends Mol. Med. 17, 347-362

7 Vander Heiden, M.G. et al. (2009) Understanding the Warburg effect: the metabolic requirements of cell proliferation. Science 324, 1029-1033

8 De Bock, K. et al. (2013) Role of PFKFB3-driven glycolysis in vessel sprouting. Cell 154, 651-663

9 Peters, K. et al. (2009) Changes in human endothelial cell energy metabolic capacities during in vitro cultivation. The role of 'aerobic glycolysis' and proliferation. Cell. Physiol. Biochem. 24, 483-492

10 Polet, F. and Feron, O. (2013) Endothelial cell metabolism and tumour angiogenesis: glucose and glutamine as essential fuels and lactate as the driving force. J. Intern. Med. 273, 156-165

11 Dranka, B.P. et al. (2010) Mitochondrial reserve capacity in endothelial cells: the impact of nitric oxide and reactive oxygen species. Free Radic. Biol. Med. 48, 905-914

12 Yeh, W.L. et al. (2008) Enhancement of glucose transporter expression of brain endothelial cells by vascular endothelial growth factor derived from glioma exposed to hypoxia. Mol. Pharmacol. 73, 170-177

13 Parra-Bonilla, G. et al. (2010) Critical role for lactate dehydrogenase A in aerobic glycolysis that sustains pulmonary microvascular endothelial cell proliferation. Am. J. Physiol. Lung Cell. Mol. Physiol. 299, L513-L522

14 Van Schaftingen, E. et al. (1982) A kinetic study of pyrophosphate: fructose-6-phosphate phosphotransferase from potato tubers. Application to a microassay of fructose 2,6-bisphosphate. Eur. J. Biochem. 129, 191-195

15 Locasale, J.W. and Cantley, L.C. (2011) Metabolic flux and the regulation of mammalian cell growth. Cell Metab. 14, 443-451

16 Buchwald, P. (2011) A local glucose-and oxygen concentration-based insulin secretion model for pancreatic islets. Theor. Biol. Med. Model. 8, 20

17 Vaupel, P. (2004) Tumor microenvironmental physiology and its implications for radiation oncology. Semin. Radiat. Oncol. 14, 198-206

18 Gatenby, R.A. and Gillies, R.J. (2004) Why do cancers have high aerobic glycolysis? Nat. Rev. Cancer 4, 891-899

19 Mertens, S. et al. (1990) Energetic response of coronary endothelial cells to hypoxia. Am. J. Physiol. 258, H689-H694

20 Pan, S. et al. (2009) Glucose 6-phosphate dehydrogenase is regulated through c-Src-mediated tyrosine phosphorylation in endothelial cells. Arterioscler. Thromb. Vasc. Biol. 29, 895-901

21 Leopold, J.A. et al. (2003) Glucose-6-phosphate dehydrogenase modulates vascular endothelial growth factor-mediated angiogenesis. J. Biol. Chem. 278, 32100-32106 
22 Vizan, P. et al. (2009) Characterization of the metabolic changes underlying growth factor angiogenic activation: identification of new potential therapeutic targets. Carcinogenesis 30, 946-952

23 Zhang, Z. et al. (2010) High glucose inhibits glucose-6-phosphate dehydrogenase, leading to increased oxidative stress and beta-cell apoptosis. FASEB J. 24, 1497-1505

24 Krutzfeldt, A. et al. (1990) Metabolism of exogenous substrates by coronary endothelial cells in culture. J. Mol. Cell. Cardiol. 22, 1393-1404

25 Favaro, E. et al. (2012) Glucose utilization via glycogen phosphorylase sustains proliferation and prevents premature senescence in cancer cells. Cell Metab. 16, 751-764

26 Lohmann, R. et al. (1999) Rat liver endothelial cell glutamine transporter and glutaminase expression contrast with parenchymal cells. Am. J. Physiol. 276, G743-G750

$27 \mathrm{Wu}$, G. et al. (2000) Glutamine metabolism in endothelial cells: ornithine synthesis from glutamine via pyrroline-5-carboxylate synthase. Comp. Biochem. Physiol. A: Mol. Integr. Physiol. 126, 115-123

28 DeBerardinis, R.J. and Cheng, T. (2010) Q's next: the diverse functions of glutamine in metabolism, cell biology and cancer. Oncogene 29, 313-324

29 Hinshaw, D.B. and Burger, J.M. (1990) Protective effect of glutamine on endothelial cell ATP in oxidant injury. J. Surg. Res. 49, 222-227

30 Unterluggauer, H. et al. (2008) Premature senescence of human endothelial cells induced by inhibition of glutaminase. Biogerontology 9, 247-259

31 Dagher, Z. et al. (2001) Acute regulation of fatty acid oxidation and amp-activated protein kinase in human umbilical vein endothelial cells. Circ. Res. 88, 1276-1282

32 Hagberg, C.E. et al. (2010) Vascular endothelial growth factor B controls endothelial fatty acid uptake. Nature 464, 917-921

33 Reihill, J.A. et al. (2011) The role of AMP-activated protein kinase in the functional effects of vascular endothelial growth factor-A and -B in human aortic endothelial cells. Vasc. Cell 3, 9

34 Elmasri, H. et al. (2009) Fatty acid binding protein 4 is a target of VEGF and a regulator of cell proliferation in endothelial cells. FASEB J. $23,3865-3873$

35 Jakobsson, L. et al. (2010) Endothelial cells dynamically compete for the tip cell position during angiogenic sprouting. Nat. Cell Biol. 12, 943-953

36 Phng, L.K. and Gerhardt, H. (2009) Angiogenesis: a team effort coordinated by notch. Dev. Cell 16, 196-208

37 Arima, S. et al. (2011) Angiogenic morphogenesis driven by dynamic and heterogeneous collective endothelial cell movement. Development $138,4763-4776$

38 Phng, L.K. et al. (2009) Nrarp coordinates endothelial Notch and Wnt signaling to control vessel density in angiogenesis. Dev. Cell 16, 70-82

39 Guarani, V. et al. (2011) Acetylation-dependent regulation of endothelial Notch signalling by the SIRT1 deacetylase. Nature 473, 234-238

40 Potente, M. et al. (2007) SIRT1 controls endothelial angiogenic functions during vascular growth. Genes Dev. 21, 2644-2658

41 Merchan, J.R. et al. (2010) Antiangiogenic activity of 2-deoxy-Dglucose. PLoS ONE 5, e13699

42 Vaisman, N. et al. (1990) Characterization of the receptors for vascular endothelial growth factor. J. Biol. Chem. 265, 19461-19466

43 Benedito, R. et al. (2009) The notch ligands Dll4 and Jagged1 have opposing effects on angiogenesis. Cell 137, 1124-1135

44 Vegran, F. et al. (2011) Lactate influx through the endothelial cell monocarboxylate transporter MCT1 supports an NF-kappaB/IL-8 pathway that drives tumor angiogenesis. Cancer Res. 71, 2550-2560
45 Sonveaux, P. et al. (2012) Targeting the lactate transporter MCT1 in endothelial cells inhibits lactate-induced HIF-1 activation and tumor angiogenesis. PLoS ONE 7, e33418

46 Ruan, G.X. and Kazlauskas, A. (2013) Lactate engages receptor tyrosine kinases Axl, Tie2 and VEGFR-2 to activate PI3K/Akt and promote angiogenesis. J. Biol. Chem. 288, 21161-21172

47 Hunt, T.K. et al. (2007) Aerobically derived lactate stimulates revascularization and tissue repair via redox mechanisms. Antioxid. Redox Signal. 9, 1115-1124

48 Fraisl, P. et al. (2009) Regulation of angiogenesis by oxygen and metabolism. Dev. Cell 16, 167-179

49 Hunt, T.K. et al. (2008) Lactate, with oxygen, incites angiogenesis. Adv. Exp. Med. Biol. 614, 73-80

50 Fraccaroli, A. et al. (2012) Visualization of endothelial actin cytoskeleton in the mouse retina. PLoS ONE 7, e47488

51 Yogurtcu, O.N. et al. (2012) A mechanochemical model of actin filaments. Biophys. J. 103, 719-727

52 Pollard, T.D. (2007) Regulation of actin filament assembly by Arp2/3 complex and formins. Annu. Rev. Biophys. Biomol. Struct. 36, 451-477

53 Romero, S. et al. (2007) How ATP hydrolysis controls filament assembly from profilin-actin: implication for formin processivity. J. Biol. Chem. $282,8435-8445$

54 Lim, K.B. et al. (2008) The Cdc42 effector IRSp53 generates filopodia by coupling membrane protrusion with actin dynamics. J. Biol. Chem. 283, 20454-20472

55 Real-Hohn, A. et al. (2010) Filamentous actin and its associated binding proteins are the stimulatory site for 6-phosphofructo-1kinase association within the membrane of human erythrocytes. Biochimie 92, 538-544

56 Baquer, N.Z. et al. (1975) Positional information and pattern regulation in hydra: enzyme profiles. J. Embryol. Exp. Morphol. 33, 853-867

57 Mitchell, B.F. et al. (2005) ATP production in Chlamydomonas reinhardtii flagella by glycolytic enzymes. Mol. Biol. Cell 16, 4509-4518

58 Hertz, L. et al. (2007) Energy metabolism in astrocytes: high rate of oxidative metabolism and spatiotemporal dependence on glycolysis/ glycogenolysis. J. Cereb. Blood Flow Metab. 27, 219-249

59 Mazzone, M. et al. (2009) Heterozygous deficiency of PHD2 restores tumor oxygenation and inhibits metastasis via endothelial normalization. Cell 136, 839-851

60 Zhang, J. et al. (2011) Angiopoietin-1/Tie2 signal augments basal Notch signal controlling vascular quiescence by inducing delta-like 4 expression through AKT-mediated activation of $\beta$-catenin. J. Biol. Chem. 286, 8055-8066

61 Eklund, L. and Saharinen, P. (2013) Angiopoietin signaling in the vasculature. Exp. Cell Res. 319, 1271-1280

62 Kitajewski, J. (2011) Endothelial laminins underlie the tip cell microenvironment. EMBO Rep. 12, 1087-1088

63 Stenzel, D. et al. (2011) Endothelial basement membrane limits tip cell formation by inducing Dll4/Notch signalling in vivo. EMBO Rep. 12, $1135-1143$

64 Cavallaro, U. and Dejana, E. (2011) Adhesion molecule signalling: not always a sticky business. Nat. Rev. Mol. Cell Biol. 12, 189-197

65 Giampietro, C. et al. (2012) Overlapping and divergent signaling pathways of N-cadherin and VE-cadherin in endothelial cells. Blood $119,2159-2170$

66 Locasale, J.W. and Cantley, L.C. (2010) Altered metabolism in cancer. BMC Biol. 8, 88

67 Curry, F.E. and Adamson, R.H. (2012) Endothelial glycocalyx: permeability barrier and mechanosensor. Ann. Biomed. Eng. 40, 828-839 


\title{
Treating Diabetes by Blocking a Vascular Growth Factor
}

\author{
Peter Carmeliet, ${ }^{1,2, *}$ Brian W. Wong, ${ }^{1,2}$ and Katrien De Bock ${ }^{1,2}$ \\ ${ }^{1}$ Department of Oncology, University of Leuven, Leuven, Belgium \\ ${ }^{2} \mathrm{VIB}$, Leuven, Belgium \\ Laboratory of Angiogenesis \& Neurovascular Link, Vesalius Research Center, B-3000, Leuven, Belgium \\ *Correspondence: peter.carmeliet@vib-kuleuven.be \\ http://dx.doi.org/10.1016/j.cmet.2012.10.015
}

\begin{abstract}
Ectopic lipid deposition in muscle and liver is associated with the pathogenesis of type II diabetes. Hagberg et al. (2012) report that targeting the vascular endothelial growth factor (VEGF)-B restores insulin sensitivity and glucose tolerance by inhibiting endothelial-to-tissue lipid transport, opening promising avenues for diabetes therapy.
\end{abstract}

Type II diabetes represents a formidable unmet medical health problem with more than 300 million people estimated to be affected worldwide. Individuals with diabetes have an elevated risk of vascular disease (atherosclerosis, stroke) and other complications. Diabetes-related deaths are expected to rise at an alarming speed over the coming years. Insulin resistance has been linked to accumulation of toxic lipid intermediates (ceramides, diacylglycerol) in skeletal muscle and liver. Hence, strategies preventing lipid deposition may offer therapeutic benefit but are not widely available (Samuel and Shulman, 2012). Hagberg et al. (2012) demonstrate that targeting the vascular endothelial growth factor $B$ (VEGF-B) restores insulin sensitivity and prevents type II diabetes by reducing lipid accumulation in muscle.

How can an angiogenic growth factor like VEGF-B be linked to diabetes? VEGF-B was one of the later members of the VEGF family to be identified, and its activity remains poorly understood (Fischer et al., 2008). It is expressed in the heart, skeletal muscle, and brown fat and binds to VEGF receptor-1 (VEGFR1; flt-1) and its coreceptor neuropilin-1 (NRP1). Genetic studies indicate that VEGF-B's angiogenic capacity is contextual and largely restricted to the heart, while pharmacological blockade inhibits ocular angiogenesis. In cancer, VEGF-B is, however, anti-angiogenic. Overall, its activity is less potent than other traditional angiogenic signals, but it induces strong arterialization of the coronary vasculature in rats (Bry et al., 2010). Importantly, VEGF-B overexpression in mouse but not in rat hearts leads to hypertrophy and signs of mitochondrial lipotoxicity, sug- gesting a possible role in cardiac metabolism (Bry et al., 2010; Karpanen et al., 2008). However, VEGF-B does not affect mitochondrial function per se (Hagberg et al., 2010; Karpanen et al., 2008). Rather, it promotes lipid transport across the endothelial barrier by upregulating the fatty acid transport proteins (FATP)3/4 (Hagberg et al., 2010). Indeed, lipid uptake and deposition in muscle were reduced, and lipids were shunted to white adipose tissue in VEGF-B-deficient mice. Consequently, the fat mass and body weight increased, and glucose uptake to the heart was enhanced. These initial observations prompted Hagberg et al. (2012) to investigate the potential of anti-VEGF-B therapy for insulin resistance.

In their follow-up study, Hagberg et al. (2012) demonstrate that genetic deficiency of VEGF-B in mouse models of insulin resistance and type II diabetes, including $d b / d b$ diabetic mice (which carry mutations in the leptin receptor gene) and mice fed a high-fat diet, reduces lipid storage in muscle, heart, and pancreas-but not liver. VEGF-B inhibition reduced plasma triglyceride and nonesterified fatty acid levels and normalized the HDL-C to LDL-C ratio, indicating a reduced risk for cardioavascular pathology. However, the body weight of VEGF-B-deficient mice on a high-fat diet was increased. Nevertheless, VEGF-B deficiency lowered blood glucose levels without increasing insulin secretion and restored insulin sensitivity and glucose uptake in the muscle and heart.

Pharmacological inhibition of VEGF-B via the administration of an anti-VEGF-B antibody to $d b / d b$ mice or rats fed a high-fat diet largely phenocopied the genetic findings and enhanced insulin sensitivity. In a preventive setting (prediabetic $d b / d b$ mice), VEGF-B blockade prevented the development of hyperglycemia, reduced lipid uptake in muscle, improved glucose tolerance, and protected against dyslipidemia. In a therapeutic setting (diabetic $d b / d b$ mice), VEGF-B inhibition halted the progression of hyperglycemia and muscle lipid uptake. In addition, VEGF-B blockade improved pancreatic islet morphology, restored insulin and glucagon expression in the islets, and reduced islet cell death in both models. In another high-fat diet rat model, coincident initiation of the high-fat diet and anti-VEGF-B treatment normalized glucose tolerance and glucose-stimulated insulin secretion, reduced glucose infusion and disposal rates in a hyperinsulinemic/euglycemic clamp study, and promoted glucose uptake in muscle-evidence of improved insulin sensitivity. These results support the idea that VEGF-B regulates lipid uptake by muscles. Given that VEGF-B regulates lipid transport across the endothelium, the findings suggest that the endothelium acts as a prominent barrier controlling muscle lipid uptake. Furthermore, blocking VEGF-B provides a mechanism to ameliorate or even prevent type Il diabetes (Figure 1).

As with any breakthrough, this study raises a number of questions. For instance, is the effect of VEGF-B on transendothelial lipid transport the only mechanism? VEGF-B has a poor angiogenic activity in healthy conditions, but an effect of this growth factor on the vasculature in conditions of insulin resistance remains possible. Another question is whether VEGF-B can control insulin signaling in 


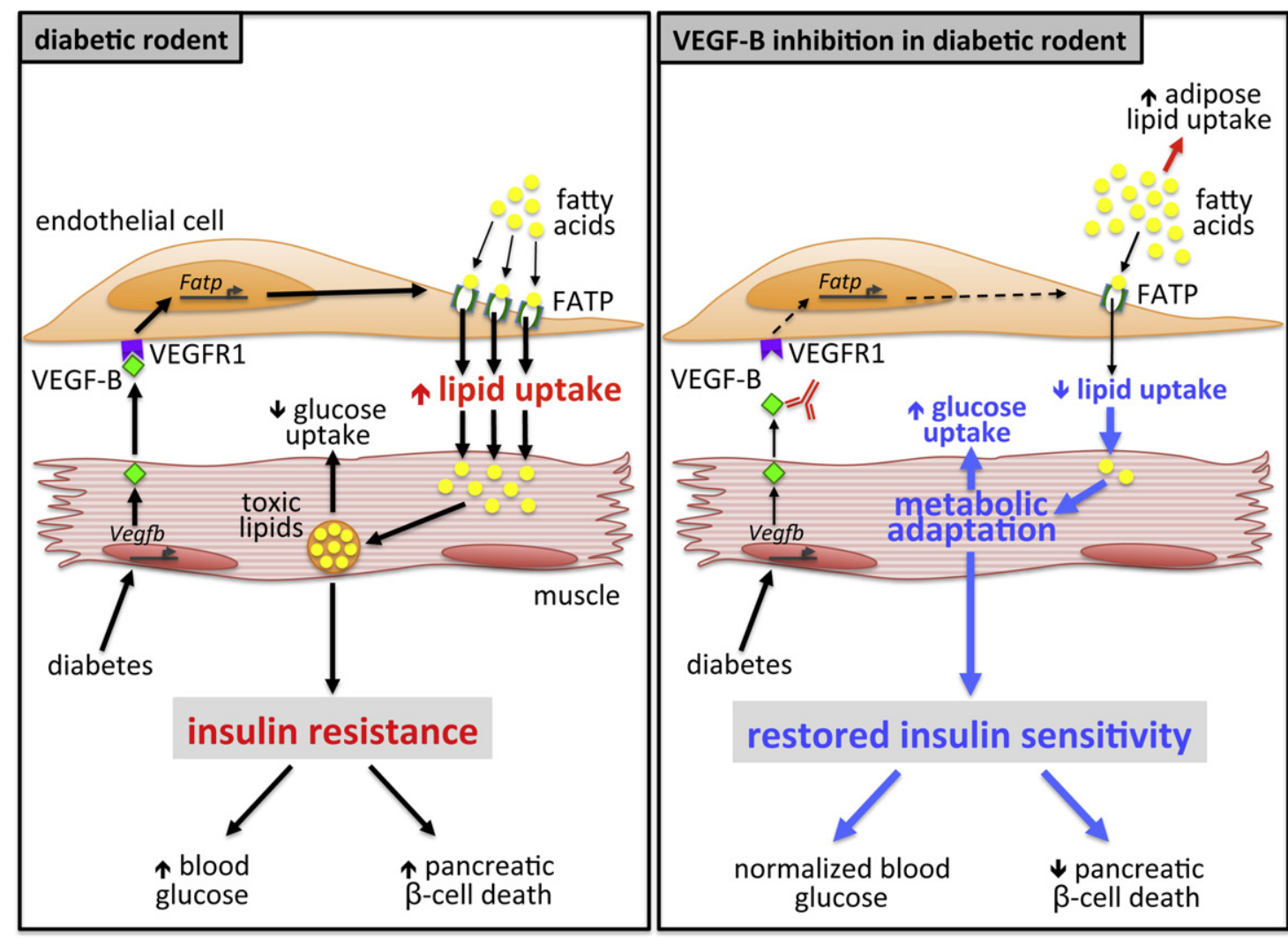

Figure 1. VEGF Promotes Diabetes by Facilitating Lipid Uptake by Muscle

In diabetic rodents (left panel), vascular endothelial growth factor (VEGF)-B, expressed in muscle, binds to VEGF receptor-1 (VEGFR1; flt-1) on endothelial cells to increase the expression of fatty acid transport proteins (FATP). FATP increase lipid transport across the endothelium to the muscle, where it accumulates as lipid droplets, resulting in insulin resistance and leading to increased blood glucose and pancreatic $\beta$ cell death. VEGF-B blockade (right panel), using a neutralizing antibody, prevents VEGF-B-mediated upregulation of FATP in the endothelium, thus reducing lipid transport to the muscle. As a result, lipid uptake in the muscle is decreased, with a compensatory increase in glucose uptake, resulting in restored insulin sensitivity and leading to normalized blood glucose and preservation of pancreatic islet area and function. Of note, peripheral adipose lipid uptake is consequently increased, leading to increased body weight. Similar data were obtained after genetic deletion of VEGF-B.

endothelial cells, a process known to increase insulin delivery to muscle (Kubota et al., 2011). Since insulin also promotes lipid uptake in muscle, VEGF-B's activity might also depend on effects on insulin. It would also be interesting to test whether endothelial deletion of NRP1 or VEGFR1, known to reduce the levels of the FATP4 transport protein in vitro (Hagberg et al., 2010), phenocopies the protective effects of VEGF-B blockade in vivo. Given that different VEGF-Bdeficient mouse strains exhibit distinct phenotypes in baseline conditions (Aase et al., 2001; Bellomo et al., 2000), a confirmation of the antidiabetic phenotype in another VEGF-B-deficient strain would strengthen the current findings. It would also be interesting to explore if VEGF-B has additional prodiabetic effects through other changes in endothelial cells, perhaps modifying endothelial cell metabolism or endothelial-to-adipocyte differentiation, or altering vascular inflammation, a process known to modify insulin sensitivity.

For anti-VEGF-B treatment to be clinically relevant, the data of the Hagberg et al. (2012) paper should be relevant for humans. While the authors paid great effort to test various rodent models, genetic association studies have not yet overwhelmingly identified VEGF-B as a possible risk factor for diabetes in humans. Another clinically relevant question is whether therapeutic blockade of VEGF-B therapy is safe. Deficiency of VEGF-B is well tolerated. Nonetheless, VEGF-B has been implicated in neurogenesis and neuroprotection (Poesen et al., 2008) and ischemic cardiac revascularization, raising the question whether VEGF-B blockade might worsen diabetic neuropathy or ischemic heart disease. The role of VEGF-B in inhibiting tumor angiogenesis will also deserve consideration. Overall, this work places the endothelium and its regulation by the VEGF-B ligand at the center stage of tissue lipid homeostasis, with promising therapeutic potential in the context of diabetes, obesity, and dyslipidemia.

\section{REFERENCES}

Aase, K., von Euler, G., Li, X., Pontén, A., Thorén P., Cao, R., Cao, Y., Olofsson, B., Gebre-Medhin S., Pekny, M., et al. (2001). Circulation 104 358-364.

Bellomo, D., Headrick, J.P., Silins, G.U., Paterson, C.A., Thomas, P.S., Gartside, M., Mould, A., Cahill, M.M., Tonks, I.D., Grimmond, S.M., et al. (2000). Circ. Res. 86, E29-E35.

Bry, M., Kivelä, R., Holopainen, T., Anisimov, A. Tammela, T., Soronen, J., Silvola, J., Saraste, A., Jeltsch, M., Korpisalo, P., et al. (2010). Circulation 122, 1725-1733. 
Fischer, C., Mazzone, M., Jonckx, B., and Carmeliet, P. (2008). Nat. Rev. Cancer 8, 942-956.

Hagberg, C.E., Falkevall, A., Wang, X., Larsson, E., Huusko, J., Nilsson, I., van Meeteren, L.A., Samen, E., Lu, L., Vanwildemeersch, M., et al. (2010). Nature 464, 917-921.

Hagberg, C.E., Mehlem, A., Falkevall, A., Muhl, L., Fam, B.C., Ortsäter, H., Scotney, P., Nyqvist, D.,
Samén, E., Lu, L., et al. (2012). Nature 490, 426-430.

Karpanen, T., Bry, M., Ollila, H.M., SeppänenLaakso, T., Liimatta, E., Leskinen, H., Kivelä, R., Helkamaa, T., Merentie, M., Jeltsch, M., et al. (2008). Circ. Res. 103, 1018-1026.

Kubota, T., Kubota, N., Kumagai, H., Yamaguchi, S., Kozono, H., Takahashi, T., Inoue, M., Itoh, S.,
Takamoto, I., Sasako, T., et al. (2011). Cell Metab. 13, 294-307.

Poesen, K., Lambrechts, D., Van Damme, P., Dhondt, J., Bender, F., Frank, N., Bogaert, E., Claes, B., Heylen, L., Verheyen, A., et al. (2008). J. Neurosci. 28, 10451-10459.

Samuel, V.T., and Shulman, G.I. (2012). Cell 148, 852-871.

\title{
Mining Genes in Type 2 Diabetic Islets and Finding Gold
}

\author{
Decio L. Eizirik ${ }^{1, *}$ and Miriam Cnop ${ }^{1,2}$ \\ ${ }^{1}$ Laboratory of Experimental Medicine, Medical Faculty \\ 2Division of Endocrinology, Erasmus Hospital \\ Universite Libre de Bruxelles (ULB), 1000 Brussels, Belgium \\ *Correspondence: deizirik@ulb.ac.be \\ http://dx.doi.org/10.1016/j.cmet.2012.10.012
}

Pancreatic $\beta$ cell failure is central in the pathogenesis of type 2 diabetes (T2D), but the mechanisms involved remain unclear. Mahdi and colleagues (2012) couple global evaluation of gene expression with coexpression network analysis of human islets from T2D patients to identify SFRP4 as an early mediator of $\beta$ cell dysfunction in T2D.

Genome-wide association studies for T2D have so far identified 65 susceptibility loci for the disease (Morris et al., 2012), but together these loci account for less than $10 \%$ of the variance in disease susceptibility. This contrasts with the situation in type 1 diabetes (T1D), where such studies have identified 50 loci across the human genome associated with T1D that explain nearly $80 \%$ of the heritability (Pociot et al., 2010). Other approaches to identify basic mechanisms of disease are therefore needed (Taneera et al., 2012). Furthermore, direct studies of the diseased human tissue-in the case of T2D, human islets are the gold standard given the central role of $\beta$ cell dysfunction in its pathogenesis - are essential to further our understanding of human diabetes (Cnop et al., 2005; Kahn, 2003). In this issue of Cell Metabolism, Mahdi and colleagues tackle these challenges by performing microarray analyses of human islets isolated from T2D and normoglycemic individuals (Mahdi et al., 2012) (Figure 1). They identify a group of T2Dassociated genes related to interleukin-1 (IL-1), a proinflammatory cytokine, and show that secreted frizzled-related protein 4 (SFRP4) is highly associated with T2D.

Previous work already suggested that $\mathrm{IL}-1 \beta$ plays a role in $\beta$ cell dysfunction and death in T1D and T2D. However, whereas a role for inflammation in $\beta$ cell loss is well established in the context of T1D (Eizirik et al., 2009), it has remained controversial for T2D (Cnop et al., 2005; Donath et al., 2008). To explain local $\mathrm{IL}-1 \beta$ production in the islets of $\mathrm{T} 2 \mathrm{D}$ individuals, one model proposed that $\mathrm{IL}-1 \beta$ production was induced by glucose, leading to upregulation of the apoptotic Fas receptor and ligand and $\beta$ cell "suicide" (Donath et al., 2008), but this was not confirmed by other groups (reviewed in Cnop et al., 2005). In recent years a more nuanced view of inflammation in T2D islets emerged, consisting of mild upregulation of cytokines and chemokines in islets from T2D patients, possibly mediated by increased circulating concentrations of the free fatty acid palmitate that induces islet IL-1 $\beta$ and TNF- $\alpha$ expression (Igoillo-Esteve et al., 2010). Experimental findings show that this metabolic "T2D-like" stress induces a mild inflammatory response, representing around $5 \%-10 \%$ of the proinflammatory response of human islets exposed to "T1D-like" conditions (Cnop et al., 2005; Igoillo-Esteve et al., 2010). The role for this "low-intensity" innate immunity-mediated inflammation in $\beta$ cell dysfunction and death in T2D remains unclear. For instance, an IL-1 receptor antagonist blocked palmitate-induced chemokine expression, but failed to prevent apoptosis (Igoillo-Esteve et al., 2010).

Using gene expression topology with weighted gene coexpression network analysis (in which coexpressed genes are clustered into gene modules based on their connectivity), Mahdi et al. now identify a T2D-related gene module enriched for IL-1-related genes. Among the most connected hub genes, the authors identify SFRP4 as highly associated with T2D, HbA1c (a measure of average glucose levels over the past 2 months), and insulin secretion (Figure 1). Subsequent functional studies then show that the SFRP4 protein is induced by the cytokine IL-1 $\beta$. Furthermore, the authors show 